\title{
SCATTERED AND INTERNAL FIELD INTENSITIES OF A HOMOGENEOUS DIELECTRIC SPHEROIDAL OBJECT ILLUMINATED WITH AN ARBITRARY FOCUSED OFF-AXIS ELECTROMAGNETIC BEAM
}

\author{
Elsayed Esam M. Khaled, and Hany L. Ibrahim ${ }^{1}$ \\ Elec. Eng. Dept., Faculty of Eng., Assiut University, Assiut, Egypt. \\ Esamk54_2000@hotmail.com ${ }^{1}$ Telecom Egypt Company, Qina, Egypt. \\ hanylouka@yahoo.com
}

(Received June 20, 2009 Accepted July 2, 2009)

\begin{abstract}
A technique combining the plane wave spectrum method and the T-matrix method is used to calculate the internal and scattered electric field intensities of a homogeneous dielectric spheroidal object illuminated with a polarized shaped beam. This technique is modified and used here for spheroidal objects of size parameter and elongation larger than those previously demonstrated in the literature. The beam is modeled using an angular spectrum of plane waves. The beam is physically realizable, satisfies Maxwell's equations and arbitrarily focused. Also the beam can be shifted far a away from the surface of the object. Scattered and internal intensities are calculated using the T-matrix method. The behavior of the internal and scattered field intensities of different spheroidal objects shows that a near resonant mode(s) can be excited in a spheroid illuminated with a focused and shifted beam that propagates in a direction perpendicular to the elongation direction of the spheroid. The axial ratio (ratio of the radii of the spheroid) is a more critical parameter for the internal and scattered field distributions in the case of an oblate spheroid as compared with a prolate case. This technique can be used to calculate the internal and scattered fields of an inhomogeneous layered spheroid illuminated with an arbitrary shaped electromagnetic beam.
\end{abstract}

\section{INTRODUCTION}

Small particles in nature such as soil particles, rain droplets, ice crystals, red blood cells and aerosols in the atmosphere, have irregular nonspherical shapes. Such particles can be approximated by spheroids [1]. Light scattering by spheroids is important in areas such as particle characterization [2], combustion, modeling nonlinear optical processes in droplets [3], aerosol detection in the atmosphere and environment [4], and biological diagnostics [5]. Other applications in nondestructive optical measurements on blood, human dermis, and soil particles need to record the light reflected from, or transmitted through those substances [2], [5]. In such cases scattering of a spheroidal object by a shaped electromagnetic beam, such as Gaussian beam is encountered.

The scattering of either an electromagnetic plane wave or a shaped electromagnetic beam by a spheroidal object is investigated by many researchers. Asano and Yamamoto [1] used the spheroidal expansion method to calculate the scattered intensity of a homogeneous spheroid (prolate or oblate) of small elongation 
and size parameter (size parameter is the ratio of the circumference of the object to the wavelength) illuminated with a plane wave. Barton et al. [6, 7] investigated the scattering of an electromagnetic Gaussian beam by a small size parameter $(\leq 15)$ and slightly elongated oblate spheroid. They used spheroidal expansion of the scalar potential of the incident fields along with the boundary matching method. They used up to fifth order correction to model the beam. Their technique gives an ill-conditioned system of equations in case of modeling a focused shifted beam scattered by a large size parameter spheroid. Although their mathematical model of the Gaussian beam does not satisfy Maxwell's equations good results were obtained because the minimum spot size of the beam was much larger than the incident wavelength and the axial ratio of the spheroid was close to 1 . Xu et al. [8] provided an approach to express the beamshape coefficients of an on-axis Gaussian beam in spheroidal coordinates. Han and Wu $[9,10]$ used the separation of variables method to obtain the angular scattering intensities of a homogeneous spheroidal particle illuminated with a Gaussian beam. Their method is limited to spheroids of size parameter $\leq 30$.

Barber and Hill [11] employed the $T$-matrix method to compute the scattered and internal intensities of axisymmetric particles such as cylinders, spheroids, and spheres of small size parameters illuminated by a plane wave. Mishchenko et al. [12] modified the convergence condition of the $T$-matrix method to enhance the calculation processes for spheroids of larger size parameters illuminated with a plane wave. They calculated only the scattered intensities. Doicu and Wriedt [13] used the plane wave spectrum method with the extended boundary condition method to calculate the angular scattering of the far field of a tightly focused Gaussian beam scattered by a spheroid of a small size parameter $(\approx 20)$. They employed the vector potential with higher-order corrections of the equivalent electromagnetic field. They obtained the beam shape coefficients with some errors.

The method we present here uses the angular spectrum of plane waves[14] to model a lowest order $\left(\mathrm{TEM}_{00}\right)$ Gaussian beam, and the $T$-matrix method [11, 15-18] to compute the electric fields inside and outside the scatterer. This combination technique was used previously $[16,17]$ to calculate only the far-field scattered by a small size parameter spheroidal object illuminated with an on-axis Gaussian beam. Also they computed the far fields for a shifted small size parameter spheroid immersed in the beam's waist. Their manipulation included re-formulation and re-orientation processes which led to complicated computations. Here we extent and reformulate the combination technique to calculate the internal and scattered intensities of a homogeneous spheroidal object (either a prolate or an oblate) of size parameter and elongation larger than those illustrated in the literature. The spheroid is illuminated with an arbitrarily on- or off-axis polarized and physically realizable Gaussian beam. The vector nature of the beam that satisfies Maxwell's equations is considered. The beam can be focused into a small spot size $(\approx$ a wavelength) shifted to a larger distances outside the object. The method can be used to model any beams including those beams having shapes deviated from their analytical representation such as those generated in laboratories (e.g. most Gaussian beams which generated experimentally have reducing oscillating tails). Moreover our technique is separable (irrelevant to the other formulation of the T-matrix [15-18]) which means that the formulation of the $T$ matrix of the scatterer is manipulated separately from the incident field modeling. The advantage of our separable technique is that: first, it deals with any physically 
realizable shaped beams, second for any particular object the elements of the $T$-matrix are calculated only once and then it can be used for different illuminations. The extension of the technique includes many parameters such as size parameter, orientation and axial ratio of the spheroid, and the shift and focusing of the beam. These parameters and their effects on the field distributions are considered. The convergence criteria are investigated for each computed case. The combination technique of the plane wave spectrum and the $T$-matrix method is applicable to a variety of objects of different shapes such as layered spheroids illuminated with an arbitrary electromagnetic beam.

Mathematical and numerical analysis are given in section II. Numerical results are presented in section III. Conclusions are outlined in section IV.

\section{NUMERICAL MODELS AND MATHEMATICAL ANALYSIS}

A homogeneous spheroidal object centered at the origin of a Cartesian coordinate system $(x, y, z)$ is considered. The radius of the spheroid along the $x$-axis is $b$ whereas that along the $z$-axis is $a$. The spheroid is oriented with respect to the coordinate system such that its semimajor axis dimension is along the $\mathrm{x}$-axis (oblate case) or along the $\mathrm{z}$ axis (prolate case). The axial ratio is $\rho=a / b(\rho<1$ for oblate case and $\rho>1$ for prolate case). The object is illuminated with a lowest order $\left(\mathrm{TEM}_{\mathrm{oo}}\right)$ monochromatic Gaussian beam (or of other different shapes) propagating in the $z$-direction as shown in Fig. 1. The beam is polarized in the $x z$-plane. Therefore the total incident electric field vector $E^{\text {inc }}$ can be expressed by[14],

$E^{i n c}(x, y, z)=E_{x}^{i n c}(x, y, z) i_{x}+E_{z}^{i n c}(x, y, z) i_{z}$,

where $i_{x}$ and $i_{z}$ are unit vectors in the $x$ and $z$ directions, respectively. The time variation $\mathrm{e}^{-\mathrm{j \omega t}}$ is omitted. We consider first the spot size of the beam at the origin, then model the beam at an arbitrary located focal point at $\left(x_{o}, y_{o}, z_{o}\right)$. We choose a Gaussian beam in which the spatial distribution of the amplitude of the transverse complex component $E_{x}$ is a Gaussian, i.e.

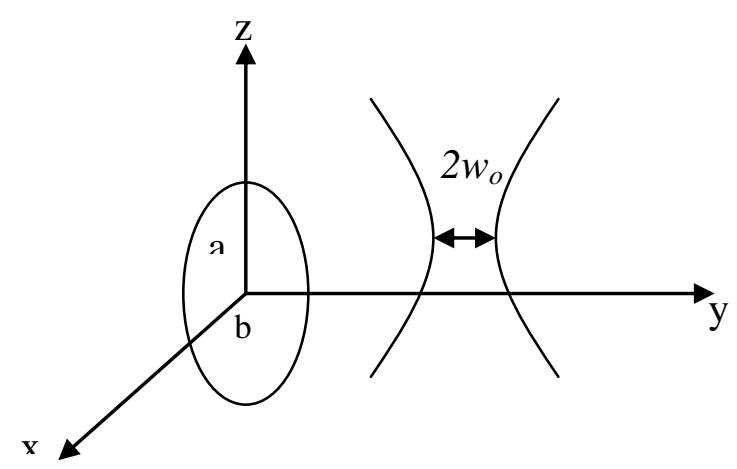

Fig. 1. A spheroidal object centered at the origin of a Cartesian coordinate system

$(x, y, z)$ of radii $a$, and $b$ along the $z$ - and $x$-axis respectively illuminated with a focused shifted Gaussian beam of a spot size $w_{o}$. 
$E_{x}^{i n c}(x, y, z)=E_{o} \frac{w_{0}}{w(z)} \exp \left[-\frac{x^{2}+y^{2}}{w^{2}(z)}\right] \cdot \exp \left[i k \frac{x^{2}+y^{2}}{2 R(z)}\right] \cdot \exp \left\{i\left[k z-\tan ^{-1}\left(\frac{z}{z_{g}}\right)\right]\right\}$,

where $E_{o}$ is the amplitude of the beam at its center, $i=\sqrt{ }-1$ and $k=2 \pi / \lambda$ is the wave number; $\lambda$ is the wavelength. The beam spot size and the radius of phase front at a distance $z$ are $\omega(z)$ and $R(z)$ respectively. The parameter $z_{g}$ is the Rayleigh length. The minimum spot size $w_{o}$ of the beam occurs at $z=0$. Also at $z=0$ the wave front is planar: $\mathrm{R}(\mathrm{z})=\infty$. After many mathematical processes and manipulations (details are specified in Reference [14]) the incident beam can be expressed in Vector Spherical Harmonics (VSH) as[14],

$$
E^{i n c}(k r)=P^{2} \sum_{m} \sum_{n} D_{m n}\left[a_{\text {emn }}^{t} M_{\text {emn }}^{1}(k r)+a_{o m n}^{t} M_{\text {omn }}^{1}(k r)+b_{\text {emn }}^{t} N_{\text {emn }}^{1}(k r)+b_{o m n}^{t} N_{o m n}^{1}(k r)\right] \text {, }
$$

where $P$, and $D_{m n}$ are normalization factors. The $m$ and $n$ are integer numbers denoting to the azimuthal mode number and the mode index respectively. The vectors $M^{l}(k r)$, and $N^{l}(k r)$ are the VSH of the first kind; and $r$ is the distance from the field point to the origin. The letters $e$ and $o$ stand for even and odd modes respectively. The coefficients $a_{e m n}^{t}, a_{o m n}^{t}, b_{e m n}^{t}$, and $b_{o m n}^{t}$ are the expansion coefficients of the incident field. The method can be applied to model any physically realizable beams which can be expressed as a sum of homogeneous plane waves even those beams having nonanalytical representation. The experimentally generated Gaussian beams are commonly having reducing oscillating tails. Although such beams have non-analytical representations they can be modeled using our technique.

The internal electric fields $E^{\text {int }}$ and scattered electric fields $E^{s}$ of a homogeneous arbitrary dielectric object illuminated with a Gaussian beam using the $T$ matrix method are [11],

$$
\begin{aligned}
& E^{\mathrm{int}}\left(\mathrm{k}^{\prime}\right)=P^{2} \sum_{m} \sum_{n^{\prime}} c_{e m^{\prime} n^{\prime}}^{t} M_{e m^{\prime} n^{\prime}}^{1}\left(\mathrm{k}^{\prime}\right)+c_{o m^{\prime} n^{\prime}}^{t} M_{o m^{\prime} n^{\prime}}^{1}\left(\mathrm{k}^{\prime}\right)+d_{e m^{\prime} n^{\prime}}^{t} N_{e m n^{\prime}}^{1}\left(\mathrm{k}^{\prime}\right)+d_{o m n^{\prime} n^{\prime}}^{t} N_{o m^{\prime} n^{\prime}}^{1}\left(\mathrm{k}^{\prime}\right) \\
& E^{S}(k r)=P^{2} \sum_{m} \sum_{n} D_{m n}\left[f_{e m n}^{t} M_{e m n}^{3}(k r)+f_{\text {omn }}^{t} M_{\text {omn }}^{3}(k r)+g_{\text {emn }}^{t} N_{\text {emn }}^{3}(k r)+g_{\text {omn }}^{t} N_{o m n}^{3}(k r)\right],
\end{aligned}
$$

where $k^{\prime}=r k \sqrt{ } \varepsilon_{r} ; \varepsilon_{r}$ is the complex dielectric constant of the scatterer relative to that of the surrounding medium. The $c_{\text {emin }}^{t}, c_{\text {om } n^{\prime}}^{t}, d_{\text {emin }}^{t}$ and $d_{\text {om } n^{\prime}}^{t}$ are the expansion coefficients of the internal electric field. The functions $M^{3}(k r)$ and $N^{3}(k r)$ are the VSH of the third kind (outgoing wave functions), and $f_{\text {emr }}^{t}, f_{\text {omn }}^{t}, g_{\text {emn }}^{t}$, and $g_{\text {omn }}^{t}$ are the scattered field expansion coefficients. In case of an axisymmetric object $m=m$. The internal and the scattered fields expansion coefficients for each azimuthal mode, $m$, are given by [11],

$$
-i\left[\begin{array}{c}
a_{\sigma}^{t} \\
b_{\sigma}^{t}
\end{array}\right]=[A]\left[\begin{array}{c}
c_{\rho}^{t} \\
d_{\rho}^{t}
\end{array}\right] \quad \& \quad\left[\begin{array}{c}
f_{\sigma}^{t} \\
g_{\sigma}^{t}
\end{array}\right]=-i[B]\left[\begin{array}{c}
c_{\rho}^{t} \\
d_{\rho}^{t}
\end{array}\right]
$$

where the A- and B-matrix are block diagonal matrices depending on the properties of 
the scatterer. Each block is a full matrix of dimension $n x n$. The indices $\sigma$ and $\rho$ are denoting to emn, omn, and emn', omn' respectively. From the matrix systems in (6) the scattered field expansion coefficients can be obtained in terms of those of the incident field as $[\mathrm{f}]=-[\mathrm{T}][\mathrm{a}]$, where $[T]=[B][A]^{-1}$ is the $T$-matrix. In case of a spherical objects the $T$-matrix is a diagonal matrix and its elements are given by $T_{l l}=B_{l l} / A_{l l}$. For spheroidal objects the $T$-matrix is a block diagonal matrix and its elements are given by $T_{l p}=\sum_{q}[B]_{l q}[A]_{q p}^{-1}$. Each element in $A$ and $B$ matrices is a surface integral over the surface of the scatterer which is calculated numerically. Note that the incident and scattered fields coefficients are expanded up to $m$ and $n$ whereas the internal field coefficients are expanded to $m$ and $n^{\prime}[11]$. Calculating the elements of the $T$-matrix for spheroids requires tests for convergence and accuracy.

\section{NUMERICAL RESULTS}

For a certain spheroidal object the T-matrix is calculated once and then it can be used for different illuminations. The equivalent spherical volume concept is considered to calculate the size parameter $x_{s}$ of the spheroid [11] that is $x_{\text {spheroid }}=x_{\text {sphere }}(a / b)^{2 / 3}$. The size parameter of the spheroid is $2 \pi a / \lambda$, where $2 a$ is the axis dimension of the spheroid along the $z$-axis. To check and confirm that the calculated internal and scattered fields are accurate we visually compared our results of the internal intensities distributions with those in Refs. [6, 7] and intensities (internal and scattered) distributions with those in Refs. [11], [14]. Also we compared computed results for angular scattering intensities with those illustrated in Refs. [9-12, 14]. No differences were noticed. When we used the software published in Ref. [19] good agreements were found only for prolate cases of size parameters $\leq 30$ either with our results or with the results of other references. For example we calculated the angular scattering intensity of a spheroid of a size parameter $x=55.930186$, refractive index $\mathrm{m}=1.3$ and an axial ratio $\rho=0.9$. The spheroid is centered at the origin and illuminated with a plane wave of a wavelength $\lambda=1.064 \mu \mathrm{m}$ propagating into the $z$-direction. Figure 2 shows the comparison between our results and those from Refs. [12] and [19]. Other computations for different cases (not shown here) illustrated the same observations.
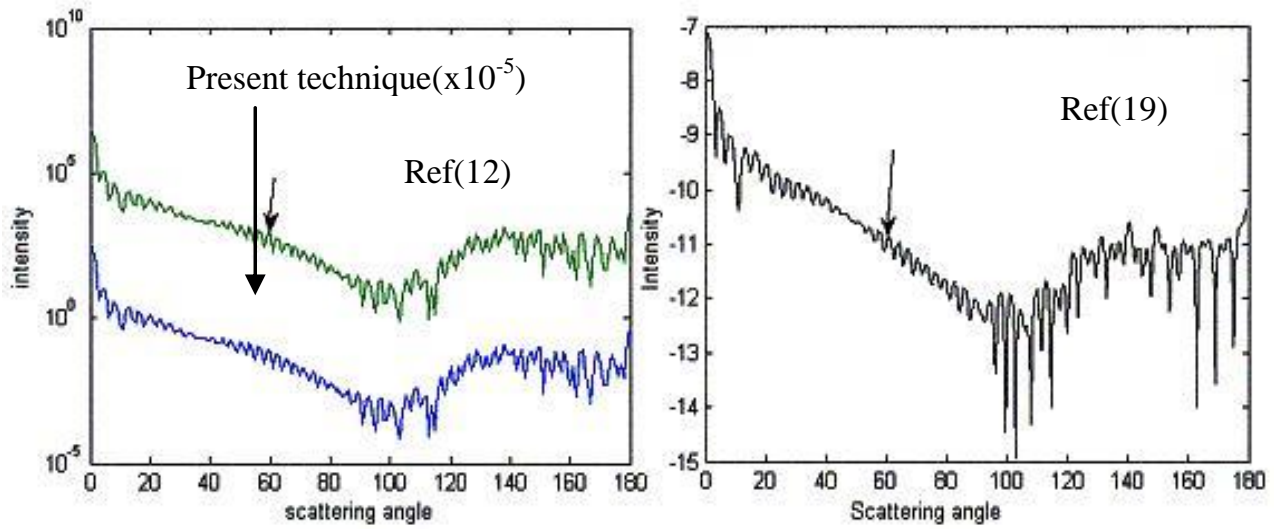

Fig. 2. Angular scattering intensity for an oblate spheroid of $\rho=0.9, x=55.930186$, and $\mathrm{m}=1.3$ illuminated with a plane wave of $\lambda=1.064 \mu \mathrm{m}$ computed using our technique and those in Refs. [12] and [19]. 
To illustrate the capabilities of our technique the intensities $E . E^{*}$ distribution inside $\left(E=E^{\text {int }}\right)$ and outside $\left(E=E^{i n c}+E^{s}\right)$ for different homogenous lossless dielectric oblate and prolate objects (in free space) are computed. First, we considered an oblate spheroid of an axial ratio $\rho=0.7143$ and a refractive index $\mathrm{m}=1.36$. The size parameter of the oblate at the operated wavelength is $x_{s}=33.79244991$ (corresponding to an equivalent spherical volume size parameter of 42.289501) and its radii are $a=5.72244251 \mu \mathrm{m}$ and $b=8.01141952 \mu \mathrm{m}$. The spheroid is illuminated with a focused Gaussian beam of a spot size $w_{o}=2 \mu \mathrm{m}$ and wavelength $\lambda=1.064 \mu \mathrm{m}$. The beam propagates along the $z$-direction. The focal point of the beam is located at the center of the oblate $\left(x_{o}=y_{o}=z_{o}=0\right)$, i.e. on-axis illumination. The contour plots of the computed intensity distribution on the $x z$-plane are shown in Fig. 3(a). Second, we considered a prolate spheroid of the same radii as the oblate spheroid but with interchanging each other i.e. $a=8.01141952 \mu \mathrm{m}$, and $b=5.72244251 \mu \mathrm{m}$. The prolate axial ratio is $\rho=1.4$ and its size parameter is $\mathrm{x}_{\mathrm{s}}=47.3094299$ (corresponding to an equivalent spherical volume size parameter of 37.8032393). All other parameters are the same as those of Fig. 3(a). The contour plot of the computed results is shown in Fig. 3(b). Figure 3 illustrates that the beam gets more focusing if it is scattered by a prolate spheroid whereas it becomes wider at its waist if it is scattered by an oblate spheroid. Moreover spots of high intensities in the internal and backscattered zones, and beam deformations are more pronounced in the oblate case. This phenomenon has important implications in studying the nonlinear processes in dielectric spheroids.

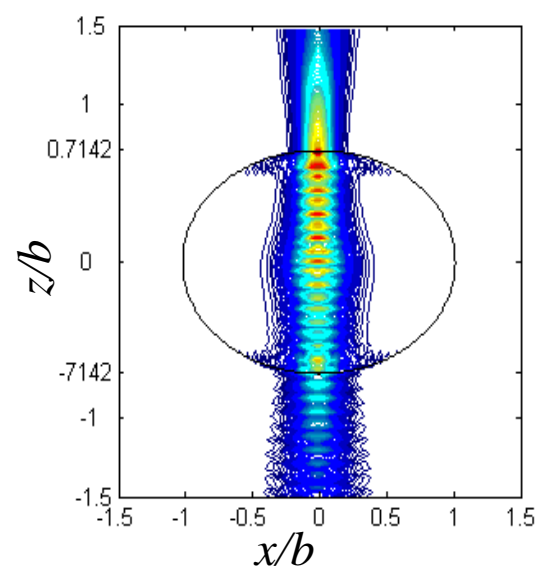

(a)

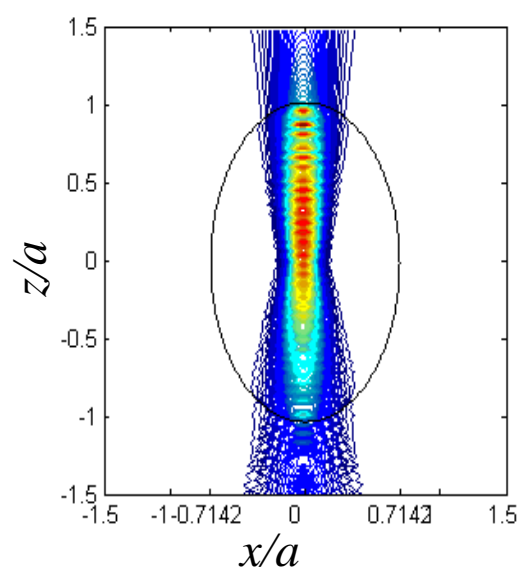

(b)

Fig. 3. Contour plots of the electric field intensity distributions in the $x-z$ plane for an on-axis Gaussian beam incident on a homogeneous spheroid of refractive index 1.36 centered at the origin. The beam spot size is $w_{o}=2 \mu \mathrm{m}$, and its wavelength is $1.064 \mu \mathrm{m}$.

(a)An oblate of an axial ratio $a / b=0.7143$ and a size parameter $x=33.79244991$. Its radii are $a=5.72244251 \mu \mathrm{m}$, and $b=8.01141952 \mu \mathrm{m}$ along the $z$ - and $x$-axis respectively. (b)A prolate of an axial ratio $a / b=1.4$ and a size parameter $x=47.3094299$. Its radii are $a=8.01141952 \mu \mathrm{m}, b=5.72244251 \mu \mathrm{m}$.

The internal and scattered intensities are calculated for two spheroids, one oblate spheroid and the other prolate spheroid having the same parameters as those of Fig. 3 except that the beam is shifted along the $\mathrm{x}$-axis by $x_{o}=b / 2=4.00570976 \mu \mathrm{m}$ for 
the oblate case, and $x_{o}=b / 2=2.86122125 \mu \mathrm{m}$ for the prolate case with $y_{o}=z_{o}=0$ for both cases. The contour plots of the computed intensity distribution on the $x z$-plane for the oblate and prolate spheroids illumination are shown in Fig. 4(a) and 4(b), respectively. Figure 4 demonstrates that the beam is tilted differently in the two cases due to the different interactions between the beam and the scatterer.

To trace the beam behavior and its interaction with the object, the beam is shifted along the $\mathrm{x}$-axis to the surface of the spheroid, that is $x_{o}=8.01141952 \mu \mathrm{m}$ for the oblate case and $x_{o}=5.72244251 \mu \mathrm{m}$ for the prolate case. All other parameters are the same as in Fig. 3. The contour plots of the calculated intensity distribution on the $x z-$ plane for the oblate and prolate are shown in Fig. 5(a) and 5(b), respectively. The results show that the electric field intensity is concentrated mainly close to the interior surface of the oblate spheroid whereas no field exists around the surface of the prolate spheroid. This distribution of the intensity inside the oblate indicates excitation of a near resonant low-order mode(s). No near resonance excitation is shown in the prolate case. More cases (not shown here) for the beam shifted farther away from the surface of an oblate and a prolate of different axial ratios are considered. All the computed results for those cases show that low-order modes can be excited in the oblate cases but no resonant modes are pronounced in the prolate spheroids. Note that the energy of a low-order morphology-dependant resonance, MDR, in a spherical dielectric object is distributed around and close to the internal surface of the sphere. As the order of the MDR gets higher its energy is distributed farther away from the surface inside the sphere [20]. If a sphere of a certain resonance size parameter is deformed to a spheroid with an axial ratio close to 1 (slightly elongated) the MDRs could be shifted in frequencies [21], [22]. Similar results as those in Figs. 4 and 5 but for plane wave illumination could be obtained approximately using the Geometrical optics method. It implements Snell's law for each ray constructing the wave of each interaction at the internal surface of the object. Since the geometrical optics does not deal with field vectors therefore it is not suitable for a focused beam illumination because it is constructed by thousands of vectors of plane waves.

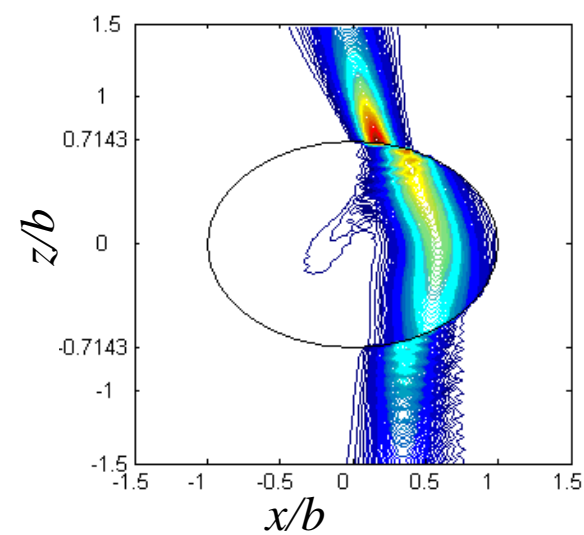

(a)

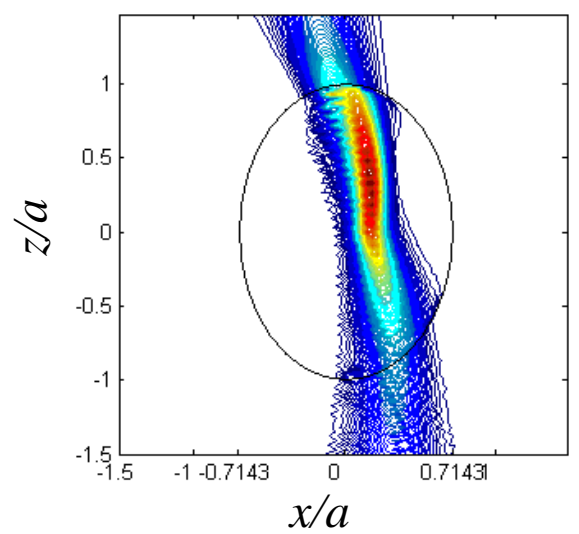

(b)

Fig.4. Contour plots of the intensity distributions in the $x-z$ plane for a spheroid. All the parameters are the same as in Fig. 3 except that the beam is shifted by $x_{o}=0.5 b$, $y_{o}=z_{o}=0$. (a)the oblate case (b) the prolate case. 


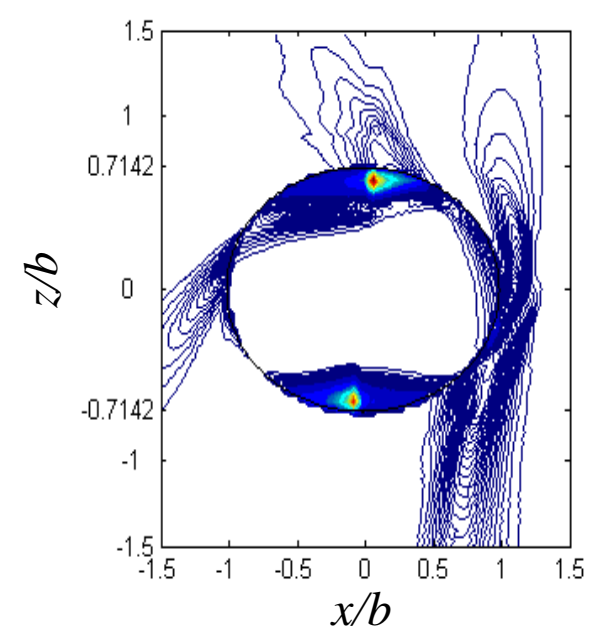

(a)

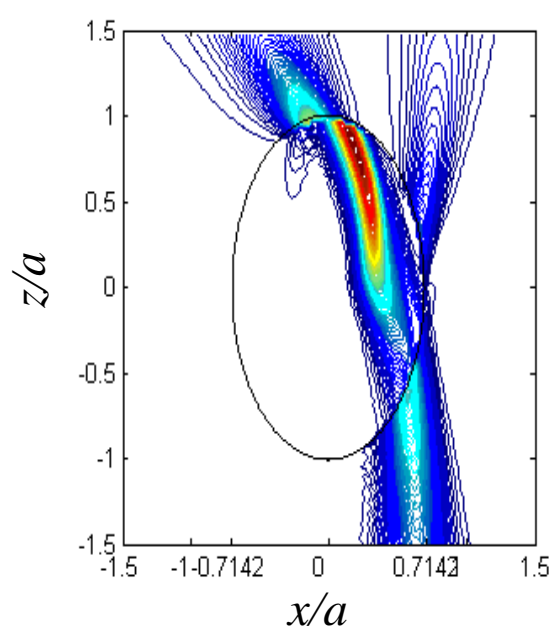

(b)

Fig.5. Contour plots of the intensity distributions in the $x-z$ plane for a spheroid. All the parameters are the same as in Fig. 3 except that the beam is shifted by $x_{o}=b, y_{o}=z_{o}=0$.

(a) the oblate case (b) the prolate case.

The effect of the axial ratio of a spheroid on the internal intensity distribution is investigated. We consider a homogeneous dielectric spherical object $(\rho=a / b=1)$ of a refractive index $\mathrm{m}=1.36$ and a resonant size parameter $x_{s}=47.3094299$ which corresponds to the $T E_{58,1} \mathrm{MDR}$. The sphere is centered at the origin and illuminated with a plane wave of a wavelength $\lambda=1.064 \mu \mathrm{m}$ and propagating in the $z$-direction. The normalized amplitudes of the internal expansion coefficients $\sum_{m} c_{e m_{n}{ }^{\prime}+} c_{o m_{n}}{ }^{\text {'for }}$ $n^{\prime}=58$ are computed. Those coefficients are corresponding to the $T E_{58,1} \operatorname{MDR}$ [23]. The computations are repeated as a function of the axial ratio with gradually increasing value ( $>1$, i.e. prolate) and with gradually decreasing value $(<1$, i.e. oblate). The computed results are shown in Fig. 6(a). Also the computations are repeated again for the same case as in Fig. 6(a) but with a Gaussian beam illumination of the same parameters as those in Fig. 3 except that the beam is shifted outside the sphere to $x_{o}=11.098098 \mu \mathrm{m}, y_{o}=z_{o}=0$. The computed results are shown in Fig. 6(b) which illustrate that the coefficient $C_{58,1}$ is maximum when $\rho=1$ (the sphere case)[23]. As the value of $\rho$ increases (prolate) or decreases (oblate) from one (sphere) the coefficient is damped for any tiny deformation. The results in Fig. 6 show that in the case of the oblate spheroid more resonant modes are pronounced than in the prolate spheroid case. The resonant modes in the oblate case have higher quality factor, Q, than those in the prolate case. Also Fig. 6 shows that more background energy exists for the plane wave illumination case since the wave illuminates the whole object. The internal energy distribution depends on, as shown here in Figs. 3-6, the size parameter, the axial ratio, the orientation of the spheroid, and on the shift of the incident focused beam. 


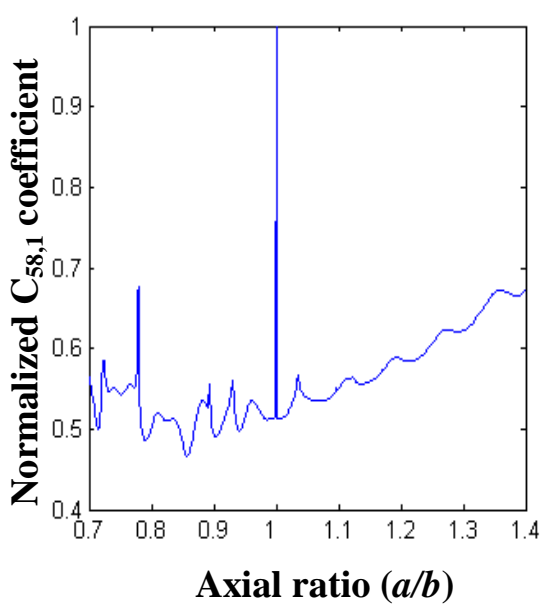

(a)

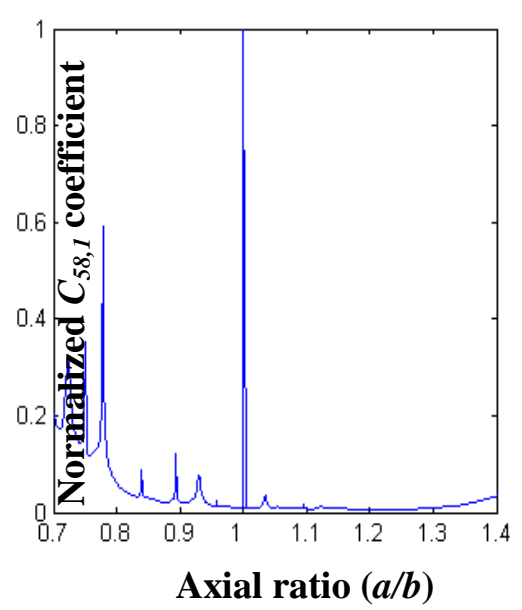

(b)

Fig. 6. Normalized amplitude of the $C_{58,1}$ coefficient as a function of the axial ratio $\rho=a / b$ of a spheroid of a size parameter $x=47.3094299$ (is a resonant size parameter of $\mathrm{TE}_{58,1}$ mode for $\rho=1$ ) and a refractive index $\mathrm{m}=1.36$, (a)a plane wave illumination with a wavelength $\lambda=1.064 \mu \mathrm{m}$ propagating in the $z$-direction, (b) a Gaussian beam illumination with parameters as those in Fig. 3 except that the beam is shifted to

$$
x_{o}=11.098098 \mu \mathrm{m}, y_{o}=z_{o}=0 .
$$

The convergence criteria are tested for each illustrated case using the formula $n_{\max }=x+4.05 x^{1 / 3}+2$ in the beginning of the calculations. Then $n_{\max }, n_{\max }^{\prime}$ are increased and the convergence is tested for each extra mode. For most cases we consider $n_{\max }=n_{\max }^{\prime}$. The summation over the azimuthal modes $m$ ranges from 0 to $n_{\max }-1$. Note that the summation over $n$ or $n^{\prime}$ is calculated for each mode $m$ (from $m+l$ to $n_{\max }$ ). Also the modes $n, n^{\prime}$, and $m$ should be tested for the Gaussian beam modeling especially for the shifted beams. For a beam with small shift number of the modes required for convergence criteria are sufficient to model the beam. Beams of larger shift require number of modes larger than those necessary for the convergence. Figure 7 shows a 1 $D$ plot of an incident non-exact Gaussian beam of $\lambda=1.064 \mu \mathrm{m}$, and $w_{o}=2 \mu \mathrm{m}$. The beam is shifted to $x_{o}=12 \mu \mathrm{m}, y_{o}=z_{o}=0$. Number of modes required to model this beam are $m=77$ and $n=78$. To model an exact Gaussian beam with the same focusing and shift more modes should be added to the computation $(m=85$ and $n=86)$.

Table (1) shows numbers of modes $n$ necessary for convergence for different spheroidal objects having the same size parameter, $x=47.3094299$, as a sphere $(\rho=1)$ of radius $a$ illuminated with a plane wave of $\lambda=2 \mu \mathrm{m}$ compared with those calculated using the criteria in Reference [12]. Note that $m=1$ for the plane wave illumination propagating in the $z$-direction.

Table (1) illustrates that number of modes $n$ necessary for convergence decreases when the radius of the sphere increases in the direction of the propagation (prolate) whereas it increases when the radius increases in the direction perpendicular to the direction of propagation. Numbers of modes $m$, and $n$ required for cases illustrated in Figs. 2 and 4, and some other cases of large size parameters are shown in Table (2). The maximum size parameter for a prolate spheroid that can be handled by our 
technique is 76.1066055 with $\rho=1.4286$. Modes necessary for convergence criteria for both the beam modeling and for the field calculations are $m=77$ and $n=78$. For an oblate spheroid the maximum size parameter is 55.930186 with $a / b=0.9$ using $m=77$ and $n=78$. These values of $m$ and $n$ get larger numbers and reach out of the limit of our codes if both the size parameter and the elongation are increased in the same time. For smaller size parameter and larger elongation the convergence occurs at smaller values of $m$, and $n$.

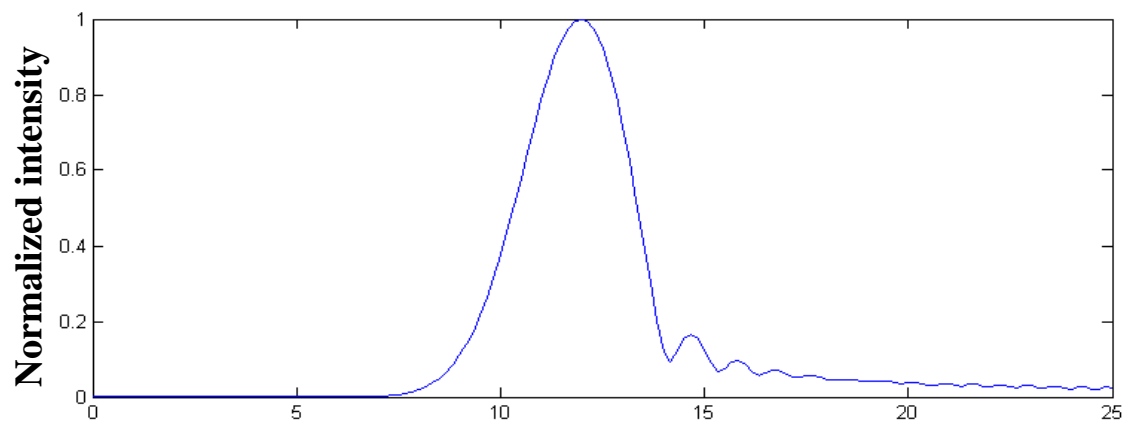

$\boldsymbol{x}$-axis

Fig. 7. A 1-D plot of an incident shifted non-exact Gaussian beam with $\lambda=1.064 \mu m$, $w_{o}=2 \mu \mathrm{m}, x_{o}=12 \mu \mathrm{m}, x_{o}=0, y_{o}=0$. Number of modes required to model the beam are $m=77$ and $n=78$.

Table (1) Numbers of modes necessary for convergence for a plane wave illumination

\begin{tabular}{|l|c|c|}
\hline \multicolumn{1}{|c|}{$a / b$} & $\begin{array}{c}\text { Present } \\
\text { technique }\end{array}$ & Reference [12] \\
\hline$a / b=1.4286$ & 54 & 60 \\
\hline$a / b=1.25$ & 58 & 59 \\
\hline$a / b=1.1111$ & 61 & 59 \\
\hline$a / b=1$ & 64 & 62 \\
\hline$a / b=0.9$ & 68 & 67 \\
\hline$a / b=0.8$ & 73 & 71 \\
\hline$a / b=0.7$ & 78 & 89 \\
\hline
\end{tabular}

Figures 3-6 demonstrate that our technique is accurate and capable to deal with cases that could not be addressed in the literature. To the best of our knowledge that the manipulation and modification of the $T$-matrix method with the plane wave spectrum technique to apply for an elongated large size parameter spheroid illuminated with considerably focused and shifted incident beam was not considered before. 
Table (2) Numbers of modes $m$ and $n$ necessary for convergence for a Gaussian beam illumination

\begin{tabular}{|l|l|l|c|c|}
\hline \multicolumn{1}{|c|}{$a / b$} & \multicolumn{1}{c|}{$x$} & \multicolumn{1}{c|}{ Shift of the beam } & $m$ & $n$ \\
\hline 1.4286 (prolate) & 76.106055 & $x_{o}=b=8.4788586$ & 77 & 78 \\
\hline 1.4 (as in Fig. 2b) & 47.3094299 & on-axis & 53 & 54 \\
\hline 1.4 (as in Fig. 4b) & 47.3094299 & $x_{0}=b$ & 53 & 54 \\
\hline 0.9 (oblate) & 55.930186 & $x_{o}=b=5.7221952$ & 77 & 78 \\
\hline 0.7143 (as in Fig. 2a) & 33.792449 & on-axis & 58 & 59 \\
\hline 0.7143 (as in Fig. 4a) & 33.792449 & $x_{0}=b$ & 58 & 59 \\
\hline 0.7143 & 47.3094299 & $x_{0}=b$ & 77 & 78 \\
\hline 0.6 & 42.682721 & $x_{0}=b$ & 77 & 78 \\
\hline
\end{tabular}

\section{CONCLUSIONS}

The technique that combining the plane wave spectrum method and the T-matrix method is used to calculate internal, and scattered electric field intensities of a homogeneous dielectric spheroidal object (a prolate or an oblate) illuminated with a focused polarized Gaussian beam. The beam is modeled by a spectrum of plane-waves. The vector nature of the beam is considered that satisfies Maxwell's equations. The beam can be arbitrarily focused and shifted to a large distance. The internal and scattered intensity distributions are computed using the T-matrix method. The combination technique is manipulated in a separate way that enables us to consider spheroids of size parameter and elongation larger than those addressed in the literature.

In the case of an on-axis illumination the beam is focused more inside the oblate but it becomes wider at its focal point inside the prolate. If the beam is shifted to or farther away from the surface of the spheroidal object along its minor axis dimension very minor and limited near resonance modes will be excited. Nevertheless near resonant low-order modes dominate the internal intensity distributions at certain values of the axial ratio if the beam is shifted to or farther away from the surface of the spheroid along the perpendicular direction of its major axis dimension.

The technique presented here is limited to spheroidal object of a size parameter up to 76.106055 with an axial ratio of 1.4286 or a size parameter 47.3094299 with an axial ratio 0.7143 illuminated with a focused incident beam shifted to a long distance outside the object.

\section{REFERENCES}

[1] S. Asano, and G. Yamamoto, "Light scattering by a spheroidal particle," Appl. Opt., 14, 29-49, 1975.

[2] S. C. Hill, A. C. Hill, and P. W. Barber, "Light scattering by size/shape distributions of soil particles and spheroids," Appl. Opt., 23, 1025-1031, 1984.

[3] M. D. Levenson, and S. S. Kano, Introduction to Nonlinear Laser Spectroscopy. Academic Press Inc., MO, USA, 1985.

[4] T. Wriedt, "A review of elastic light scattering theories," Part. Syst. Charact., 15, 67-74, 1998. 
[5] A. M. K. Nilsson, P. Alsholm, and S. Andersson-Engels, "T-matrix computations of light scattering by red blood cells," CODEN: LUTEDX/(TEAT-7068)/1-24/(1988), Department of Electroscience, Electromagnetic Theory, Lund Institute of Technology, Sweden, August 8, 2001.

[6] J. P. Barton and D. R. Alexander, "Electromagnetic fields for an irregularly shaped, near spherical particle illuminated by a focused laser beam," J. Appl. Phys., 69, 7973-7986, 1991.

[7] J. P. Barton, "Internal and near-surface electromagnetic fields for a spheroidal particle with arbitrary illumination," Appl. Opt., 34, 5542-5551, 1995.

[8] F. Xu, K. Ren, and X. Cai, "Expansion of arbitrarily oriented, located and shaped beam in spheroidal coordinates," J. Opt. Soc. Am., A14, 2006.

[9] Y. Han, and Z. Wu, "Scattering of a spheroidal particle illuminated by a Gaussian beam," Appl. Opt., 15, 40, 2001.

[10]Y. Han, and Z. Wu, "Absorption and scattering by an oblate particle," J. Opt. Soc. Am., A4, 74-77, 2002.

[11]P. W. Barber, and S. C. Hill, Light Scattering by Particles: Computational Methods. World Scientific, Singapore, 1990.

[12]M. I. Mishchenko, L. D. Travis, and A. A. Lacis, Scattering, Absorption, and Emission of Light by Small Particles. NASA Goddard Institute for Space Studies, New York, 2005.

[13]A. Doicu and T. Wriedt, "Plane wave spectrum of electromagnetic beams," Optics Communications, 136, 114-124, 1997.

[14]E. E. M. Khaled, S. C. Hill, and P. W. Barber, "Scattered and internal intensity of a sphere illuminated with a Gaussian beam," IEEE Trans., Antennas Propag., Ap-41, 295-303, 1993.

[15]P. Barber and C. Yeh, "Scattering of electromagnetic wave by arbitrarily shaped dielectric bodies," Appl. Opt., 14, 2864-2872, 1975.

[16]S. Colak, C. Yeh, and L. W. Casperson, "Scattering of focused beams by tenuous particles," Appl. Opt., 18, 294-302, 1979.

[17] C. Yeh, S. Colak, and P. Barber, "Scattering of a sharply focused beams by arbitrarily shaped dielectric particles: An exact solution," Appl. Opt., 21, 44264433, 1982.

[18]P. W. Barber, "Differential-scattering of electromagnetic wave by homogeneous isotropic dielectric bodies," Ph.D. dissertation, University of California, Los Angeles, 1973.

[19]Y. Han, Gaussian Beam Scattering by Spherical and Spheroidal Particle Software. Department of Physics, School of Science, Xidian University, Germany, 2007.

[20]S. C. Hill and R. E. Benner, "Morphology-dependant resonances associated with stimulated processes in microspheres," J. Opt. Soc. Am., B3, 1509-1514, 1986.

[21]M. M. Mazumder, S. C. Hill, and P. W. Barber, "Morphology-dependant resonances in inhomogeneous spheres: Comparison of layered T-matrix and timeindependent perturbation method," J. Opt. Soc. Am., A9, 1844-1853, 1992.

[22]P. W. Barber and S. C. Hill, "Effects of particle nonsphericity on light scattering," Proceedings of the International Symposium on Optical Sizing: Theory and Practice, Rouen, France, May 12-15, 1987. 
[23]E. E. M. Khaled, S. C. Hill, and P. W. Barber, "Internal Electric energy in a spherical particle illuminated with a plane wave or off-axis Gaussian beam," Appl. Opt., 33, 524-532, 1994.

\section{شدة المجالات المشتته داخل وخارج جسم بيضاوي متجانس ومن مادة عازلة ومسلط عليه شعاع كهرومغناطيسي أختياري التركيز وذو ازاحة}

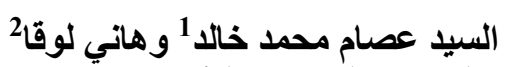

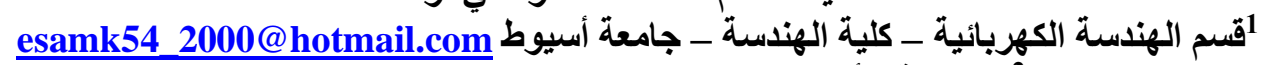

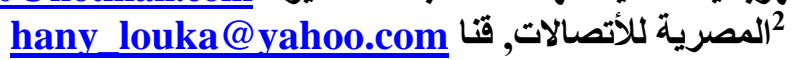

تم تطويرأسلوب لحساب شدة المجالات الكهربية داخل وخارج جسم بيضاوي متجانس ومن مادة

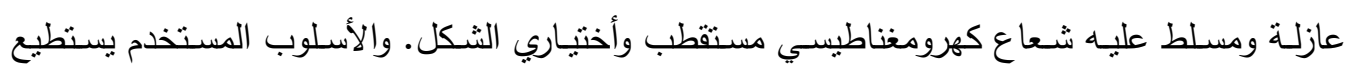

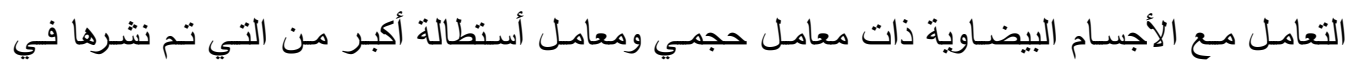
الأبحاث من قبل. وهذا الأسلوب يعتمد على طريقة تحليل الأشعة المسلطة الى موجات مستوية قاعدية

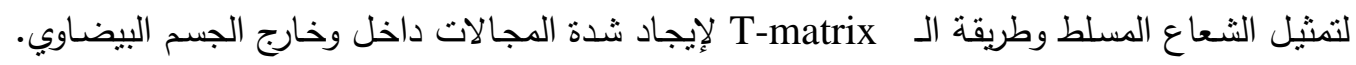

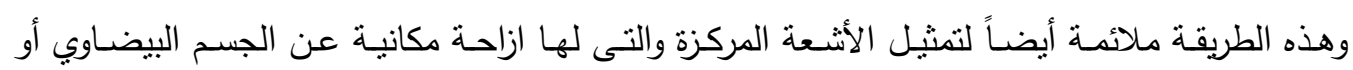

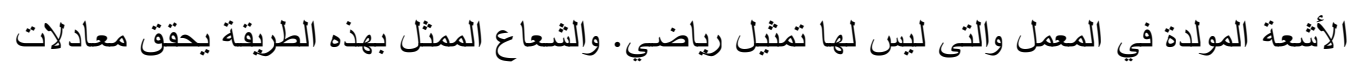
ماكسويل.

من حساب دراسة شدة المجالات داخل الجسم البيضاوي أظهرت أنه من الممكن توليد موجات

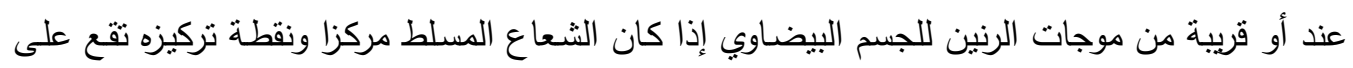

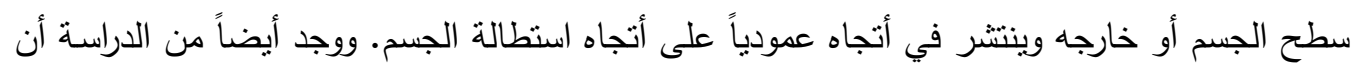

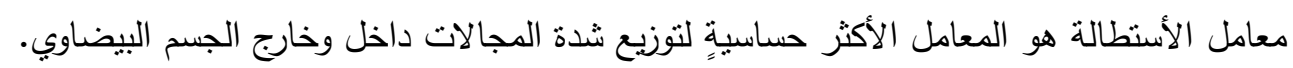

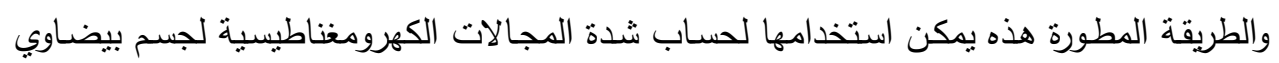
غير متجانس قطرياً أي ذات طبقات متحدة المركز ومسلط عليه شعاع كهرومغناطيسي مركز ذات شكل أختياري وذات أزاحة بالنسبة للجسم. في ذات طبنات 